\title{
Micropatterning of hybrid PDMS for replica leaves
}

\author{
Michal Bernach ${ }^{1,2 *}$, Rebecca Soffe ${ }^{2}$, Mitja N. P. Remus-Emserman ${ }^{1}$ and Volker Nock ${ }^{2 *}$ \\ ${ }^{1}$ School of Biological Sciences, University of Canterbury, Christchurch, New Zealand \\ ${ }^{2}$ Department of Electrical and Computer Engineering, University of Canterbury, \\ Christchurch, New Zealand \\ E-mail: michal.bernach@pg.canterbury.ac.nz,volker.nock@canterbury.ac.nz
}

\begin{abstract}
Artificial surfaces representing key features of plant leaves, such as surface topography and nutrient availability, promise to play an important role in phyllosphere microbiology. While polydimethylsiloxane (PDMS) constitutes an ideal candidate for leaf replica materials, its inherent hydrophobicity limits the diffusion of aqueous liquids. To solve this, we added superabsorbent polymer fillers Carbopol ${ }^{\circledR}$ and Permulen ${ }^{\mathrm{TM}}$ to PDMS, to increase the diffusion of aqueous liquids. We demonstrate that hybrid PDMS retains its capability to resolve leaf microstructures, while exhibiting similar contact angles to PDMS and the abaxial surface of Arabidopsis thaliana leaves. Water diffusion and mass flow through hybrid PDMS membranes were consistently higher than PDMS over the $144 \mathrm{~h}$ experimental duration. In addition, bacteria distribution patterns on hybrid PDMS were similar to those observed on A. thaliana leaves. However, the patterns observed on hybrid PDMS appeared to be influenced by the increase in wettability. These findings demonstrate that hybrid PDMS replicas can be used as an artificial surface to study bacterial behaviour and microbe-microbe interactions.
\end{abstract}




\section{Introduction}

In recent years, microbial life of the above ground parts of plants, the phyllosphere, has gained significant attention. The growing interest in this area is due to the need to understand how changes within the phyllosphere microbiome, impact plant health and consumer wellbeing. Plant leaves, are the most prominent elements of the phyllosphere and their surfaces are widely colonised by epiphytic microorganisms. ${ }^{1,2)}$ Leaves can also be considered a model habitat for phyllosphere microbiology studies. ${ }^{1,3)}$

Artificial surfaces enable a reductionist approach, which allows the investigation of plant-microbe and microbe-microbe interactions to be studied indivdually. ${ }^{4)}$ Flat artificial surfaces, that lack spatial heterogeneity, such as nutrient agar, are routinely used in phyllosphere microbiology. Such surfaces have enabled the identification and isolation of microbial inhabitants, provided information on their diversity, and approximate location on the leaf surface. ${ }^{5-7)}$ Inert materials, such as metals, glass or other inorganic materials, have been used in studies investigating bacterial attachment processes. ${ }^{8-10)}$

A number of the microstructured artificial surfaces have been used in phyllosphere microbiology studies. For instance, reconstituted cuticular wax, which closely resembles the nanometer crystalline patterns of leaves has been used to compare the influence of different wax compositions on the establishment of bacterial communities. ${ }^{11)}$ In addition, enzymatically isolated leaf cuticles have been used to determine the rates of water and sugar permeation to the surface, where they become available to epiphytes. ${ }^{12,13)}$ Furthermore, microfabricated silicon surfaces were used to investigate the influence of different structures on bacterial surface attachment. ${ }^{14)}$

The above-mentioned studies highlight the importance of using artificial surfaces for answering questions relating to the fundamental understanding of phyllosphere microbiology. Current artificial surfaces, however, are not representative of the complex leaf surface topography and physicochemistry. Biomimetic replica leaves are an important development that has the potential to mimic this complexity. ${ }^{4)}$ The majority of biomimetic replica leaves fabricated thus far, have replicated the superhydrophobic, superoleophobic, and superhydrophilic properties of leaves. These properties have been applied in producing anti-reflection, anti-fouling, and anti-fogging coatings. ${ }^{15-19)}$ Yet, some studies using biomimetic replica surfaces for phyllosphere microbiology applications are starting to appear. An example can be found in the study conducted by Zhang et al., where they report the influence of replicated leaf features on the behaviour of Escherichia coli on agarose replica spinach leaves. ${ }^{20)}$ 
Recently, we have shown that the elastomer polydimethylsiloxane (PDMS) is more suitable to replicate leaf surfaces than other materials, such as agarose and gelatin. We compared a number of parameters including: the resolution, degradation, contact angles, and bacterial survival on the aforementioned materials. ${ }^{21)}$ Moreover, we have also established a versatile protocol, which enables the replication of the inherently fragile Arabidopsis thaliana leaves. A. thaliana is a model system for molecular plant genetics, molecular plant biology, and microbial ecology. ${ }^{22)}$

In this study, we focus on increasing the permeability of PDMS to water to produce a controllable flow of water and nutrients to the replica leaf surface. The supply of nutrients in particular, is essential for the continuous cultivation of microorganisms on an artificial surface. To this extent we enriched PDMS with superabsorbent polymers - Carbopol ${ }^{\circledR}$ and Pemulen ${ }^{\mathrm{TM}}$ to produce a superabsorbent PDMS polymer blend - hybrid PDMS. Such fillers were shown by Borde et al. to increase water diffusion through PDMS films. ${ }^{23)}$ Carbopol is a polyacrylic acid base polymer, while Pemulen is a copolymer combining acrylic acid and C10-C30 alkyl acrylate. Here we report a fabrication protocol for producing microstructured hybrid PDMS surfaces, which contain 5\% of the aforementioned fillers. We examined the fidelity of the hybrid PDMS surfaces, using 3D optical profilometer imaging. Additionally, we confirmed that the addition of fillers increased the diffusion of water through PDMS. Moreover, we compared identical replica leaf surfaces of replicas produced in PDMS, and hybrid PDMS using optical microscopy.

. Finally, we assessed if the addition of fillers influenced the surface hydrophobic properties of the replica leaves, or the bacterial distribution patterns of - Pantoea agglomerans 299R, a model microorganism for leaf colonisation, on our replica leaf surfaces.

\section{Experimental methods}

\subsection{Plant growth}

Arabidopsis thaliana Columbia-0 (Col-0) wild-type plants were grown in plastic pots $(70 \times 70 \times 90 \mathrm{~mm})$. Two thirds of the pots were filled with potting mix containing 9 monthcontrolled release fertiliser (Canterbury Landscape, New Zealand). Two grams of Insect Lawngard Prills (KiwiCare, New Zealand) was sprinkled on top of the potting mix. To fill the pot a layer of sieved potting mix was added, using a $5 \mathrm{~mm}$ mesh sieve. Prior to being sown, A. thaliana Col-0 seeds were suspended in water and stratified for two days in the dark at $4{ }^{\circ} \mathrm{C}$. The plants were grown on raised trays with holes, which enabled the gradual absorption of water. For the first three weeks of cultivation, plastic covers were placed over 
the plant trays. The covers contained holes to enable gas exchange. The plants were grown inside a growth chamber (Contherm Precision Environmental Chamber, Contherm Scientific, New Zealand) in long day conditions: $16 \mathrm{~h}$ of light at $21^{\circ} \mathrm{C}$ and $8 \mathrm{~h}$ of darkness at $18{ }^{\circ} \mathrm{C}$. The plants were watered once a week.

\subsection{PDMS patterned template fabrication}

A PDMS template was produced from a negative-tone photoresist mold master, which was fabricated using a standard soft-lithography processes described previously. ${ }^{21)}$ Briefly, the PDMS (Sylgard 184, Dow Corning) was prepared at a ratio of 10:1 w/w (base:curing agent), was degassed in a vacuum desiccator (Z119016, Sigma-Aldrich) until no air bubbles were visible. The mixture was then poured onto the mold master containing an array of circular pillars of $5 \mu \mathrm{m}$ in height and a diameter of $15 \mu \mathrm{m}$. In order to remove any introduced air bubbles, the PDMS was thoroughly degassed for a second time. Afterwards, the PDMS and mold master were placed on a $80^{\circ} \mathrm{C}$ hot plate for two hours to polymerize the PDMS. Then, the PDMS template was peeled of the mold master. Prior to the application of either PDMS or hybrid PDMS, the template was treated with either 1) $0.3 \% \mathrm{w} / \mathrm{v}$ hydroxypropylmethylcellulose (HPMC; H8384, Sigma-Aldrich) in a phosphate buffer saline (PBS, P4417, Sigma-Aldrich) for 10 minutes ${ }^{24,25)}$, or with 2) oxygen plasma followed by trichloro(1H,1H,2H,2H-perfluorooctyl)silane (448931, Sigma-Aldrich) treatment. ${ }^{26,27)}$ During either treatment, a thin anti-adhesive layer was deposited onto the surface. This layer enables patterned PDMS to be easily peeled off the PDMS template.

\subsection{Leaf imprint fabrication}

A. thaliana leaf imprints were fabricated as previously described. ${ }^{22)}$ Briefly, the leaves were first washed with deionised water, divided into smaller pieces and affixed to the bottom of a Petri dish with double-sided tape (Orabond 1397PP, ORAFOL Europe GmbH, Germany). In order to flatten the leaves, these were gently pressed onto the double-sided tape using Parafilm "M" (Bemis, USA). The leaves were then placed in an automatic desiccator (Secador 2.0, SP Scienceware Bel-Art, USA) until the residual moisture on the leaf surfaces had evaporated. PDMS was prepared at ratio of 20:1 w/w, mixed thoroughly and degassed in a vacuum desiccator for 30 minutes. Afterward, the PDMS was poured onto the leaves and degassed again for approximately one hour. In order to cure the PDMS, the Petri dish was placed in a $45^{\circ} \mathrm{C}$ oven for $20 \mathrm{~h}$. After removing the Petri dish from the oven, the Petri dish was left to cool to room temperature, and the leaf imprints were peeled off the affixed leaves. Any remaining leaf debris was removed using a digestion solution consisting of $3.5 \% \mathrm{w} / \mathrm{v}$ sodium hydroxide $(\mathrm{NaOH}, \mathrm{S} 5881$, Sigma-Aldrich) and $2.5 \% \mathrm{w} / \mathrm{v}$ sodium 
carbonate $\left(\mathrm{Na}_{2} \mathrm{CO}_{3}, 222321\right.$, Sigma-Aldrich) dissolved in deionized water. ${ }^{28)}$ The solution was placed on a $160^{\circ} \mathrm{C}$ hot plate and stirred (SP88857105, Cimarec + , Thermo Scientific), until all components were dissolved. The PDMS leaf imprints were placed in the digestion solution for 20 minutes, then in deionized water for 20 minutes, followed by rinsing with deionized water and drying with dry nitrogen. Prior to the following fabrication processes, the PDMS template was treated with oxygen plasma for $60 \mathrm{~s}$ (Tergeo Plasma Cleaner, PIE Scientific USA), followed by trichloro $(1 \mathrm{H}, 1 \mathrm{H}, 2 \mathrm{H}, 2 \mathrm{H}$-perfluorooctyl)silane treatment under vacuum for an hour.

\subsection{Membrane mold fabrication}

A 4" prime grade silicon wafer was used as a substrate for the membrane mold master. To prepare the wafer for the application of the dry film photoresist, it was first placed in a $185^{\circ} \mathrm{C}$ overnight to remove any moisture. The wafer was then removed from the oven and left to cool to room temperature. To clean the wafer, it was placed in oxygen plasma at $100 \mathrm{~W}$ for 10 minutes. Photolithography on negative-tone dry film resist (SUEX D100, DJ MicroLaminates) was used ${ }^{29)}$ to produce a mold master with a height of $100 \mu \mathrm{m}$, and circular wells with a diameter of $22 \mathrm{~mm}$.

\subsection{Patterned materials fabrication}

To fabricate pure patterned PDMS (referred to as PDMS in the following) for comparison, a ratio of 10:1 w/w was used. To prepare the hybrid PDMS, the fillers Carbopol (971P NF, Lubrizol) or Pemulen (TR-2 NF, Lubrizol) were added to the base and thoroughly mixed. Following this, the curing agent was added and all components were thoroughly mixed. The final concentration of the fillers was 5\% w/w. The PDMS or hybrid PDMS was degassed in a vacuum desiccator for 30 minutes. After the PDMS or hybrid PDMS was poured onto the PDMS template, they were degassed again for an hour. For curing the PDMS template covered with either PDMS or hybrid PDMS was placed on an $80^{\circ} \mathrm{C}$ hot plate for two hours. Afterwards, the patterned PDMS or hybrid PDMS was gently peeled of the template.

To fabricate the leaf replicas, PDMS and hybrid PDMS were prepared as described above. After pouring onto the leaf imprint, either was degassed again for an hour. In order to cure, the leaf imprints were placed on an $80^{\circ} \mathrm{C}$ hot plate for two hours and replica leaves gently peeled of the template after baking.

To fabricate the PDMS and hybrid PDMS membranes, exclusion molding technique was used. After degassing, the PDMS or hybrid PDMS was poured onto the mold and covered with an acetate sheet (overhead projector transparency film, OfficeMax). A 4" Pyrex wafer and rubber sheet were placed on top, to balance the distribution of the further added weight 
$(\sim 200 \mathrm{~g})$. The set-up was then placed on an $80{ }^{\circ} \mathrm{C}$ hot plate for two hours. The membranes of $\sim 150 \mu \mathrm{m}$ thickness were then manually peeled off the mold.

\subsection{Optical profilometry and visualisation of the replica surface features}

To acquire the 3D (three-dimensional) optical profiles a Profilm3D optical profilometer (Filmetrics Inc., USA), equipped with a 20× objective (CF Plan 20×/0.40 DI, Nikon) was used. The optical profiles of the patterned materials were processed using the Profilm3D software 2018 (ver. 3.2.7.2, Filmetrics Inc., USA). Invalid pixels were filled in and all images were flattened with the built-in 3-point level function to compensate for the nonleveled placement of the samples.

The PDMS or hybrid PDMS replica leaf fidelity was assessed using bright field microscopy on an optical microscope (BX30, Olympus), equipped with a $20 \times$ objective (Olympus) and a digital camera (DFC295, Leica). IrfanView image processing software (v.4.51) was used to capture images, which were then processed with Fiji (version $1.52 \mathrm{~h}){ }^{30}{ }^{3}$

\subsection{Contact angle measurement}

To conduct the contact angle measurements, we used a goniometer (CAM200, KSV Instruments Ltd, Finland) integrated with KSV CAM Optical Contact Angle and Pendant Drop Surface Tension Software (ver. 4.01). Contact angle measurements were taken for the abaxial surface of the living leaf and replicas. Five samples were measured for A. thaliana and each of the replica compositions. All samples were $11.5 \mathrm{~mm}$ in diameter. Droplets of deionized water of volumes less than $80 \mu \mathrm{L}$ were recorded.

\subsection{Permeability measurement}

The thickness of the PDMS and hybrid PDMS membranes were measured using a micrometer screw. To measure the permeability of the materials to deionized water, the setup described by Remus-Emsermann et al. was used. ${ }^{13)}$ Membranes (16 mm diameter) were immobilized between two of stainless steel diffusion chambers. A single layer of aluminium foil (16 mm diameter; Ullrich Aluminium) was used as negative control. The surface of the diffusion chambers components that were in contact with the membranes or each other were covered with high-vacuum silicone grease to limit the leakage of deionized water. After sealing the diffusion chambers, deionized water was added to the donor chamber. The chambers were then placed in container with silica gel overnight in $28^{\circ} \mathrm{C}$, with membranes facing upwards, to remove any surface humidity. Following this, the weight of the chambers was measured using a pipette calibration balance (AND-Australia, AD-4212A-PT, 110g / $0.1 \mathrm{mg}$ Pipette Calibration Balance / Accuracy Tester). The weight of the chambers were recorded every $24 \mathrm{~h}$ for six days, to determine the water diffusion through the membranes. 
The measured water diffusion values adjusted for slight variances in the membrane thicknesses, given by equation 1 :

$$
W_{\text {normalised }}=\frac{t_{\text {membrane }}}{t_{\text {membrane-set }}} W_{\text {measured }}
$$

Where, $t_{\text {membrane }}$ is the thickness of the membrane $(\mathrm{m}), \mathrm{t}_{\text {membrane-set }}$ is the specified membrane thickness of $150 \mu \mathrm{m}$, and $\mathrm{W}_{\text {normalised }}$ and $\mathrm{W}_{\text {measured }}$ are the normalised water diffusion $(\mathrm{g})$ and measured water diffusion $(\mathrm{g})$ values, respectively.

The mass flux values were calculated as mass $(\mathrm{g})$ that diffused through a membrane (1.13 $\mathrm{cm}^{2}$ diameter of exchange area) per second. For each experiment, three samples for each sample was measured.

\subsection{Bacteria Pantoea agglomerans 299R::MRE-Tn7-145 culture protocol}

A red fluorescently-labelled strain of the phyllosphere model organism - Pantoea agglomerans 299R (P. agglomerans 299R::MRE-Tn7-145) was grown overnight on nutrient agar plates (13 $\mathrm{gL}^{-1}$ nutrient broth and $15 \mathrm{gL}^{-1}$ bacteriological Agar, Oxoid) supplemented with $20 \mathrm{mg} \mathrm{L}^{-1}$ of gentamicin (Duchefa) at $30^{\circ} \mathrm{C} .{ }^{31,32)}$ The bacteria was harvested with a sterile inoculation loop and suspended in $5 \mathrm{~mL}$ of sterile phosphate buffer saline (PBS, $\mathrm{pH}$ 7.4, P4417, Sigma-Aldrich). Bacteria was then washed twice by centrifugation at $1150 \mathrm{RCF}$ for five minutes at $10{ }^{\circ} \mathrm{C}$ and resuspension in $5 \mathrm{~mL}$ PBS. The supernatant was removed and the bacteria was resuspended in PBS to an OD600 of 0.7 (approximately $7 \times 10^{8}$ bacteria per $\mathrm{mL})$.

\subsection{Bacteria visualisation}

PDMS and hybrid PDMS replica leaves were sterilised with ultraviolet radiation for 15 minutes. Afterwards, the replica leaves were placed aseptically inside a vacuum desiccator (Z119016, Sigma-Aldrich) for two hours. Following this, replica leaves were inoculated with two $200 \mu \mathrm{l}$ aliquots of P. agglomerans 299R::MRE-Tn7-145 bacterial solution using an airbrush (Kkmoon T-180 Airbrush, China) at $1 \times 10^{5} \mathrm{~Pa}^{33)}$ Images of $P$. agglomerans 299R::MRE-Tn7-145 distributions on the PDMS and hybrid PDMS replica leaves were taken using a Nikon Eclipse 80i fluorescence microscope, equipped with G-2A and BF filters (Nikon), LED illumination (X-Cite 120 LED Boost, Excelitas Technologies), a digital CMOS camera (C11440-22CU ORCA-Flash 4.0 V2, Hamamatsu Photonics), 20× objective (LU Plan Fluor 0.45, Nikon) and HC Image software (v. 4.2.0.33, Hamamatsu Corporation).

\section{Results and discussion}




\subsection{Replication using hybrid PDMS}

The microfeatures present on the plant leaf surface are known to affect microbial communities on the surface. ${ }^{33,34)}$ Therefore, it is important to replicate the microfeatures into any artificial surface used for phyllosphere microbiology, including hybrid PDMS, at the highest possible detail. Previously, we have demonstrated that PDMS is the most suitable material to replicate the surface of a plant leaf. ${ }^{21)}$ The elastomer was shown to have replication capabilities to reproduce complex microstructures on the leaf surface, including stomata and trichomes (leaf hairs). At the same time it exhibited material degradation, surface energies, and bacterial survival properties comparable to isolated leaf cuticles, thus making PDMS an ideal candidate for the production of replica leaf surfaces. At the same time, we identified a major drawback of PDMS, namely its low permeability to aqueous solutions, such as the liquid nutrients and general moisture, typically supplied by a leaf to its surface to encourage and guide bacterial colonization. ${ }^{32)}$ One way to overcome this limitation of PDMS, while retaining its other material properties, is the addition of superabsorbent polymer fillers. A previous study by Borde et al. characterized the effect of such fillers via water transport studies, swelling tests, scanning electron microscopy imaging, and confocal microscopy. ${ }^{23)}$ They found that cross-linked superabsorbent polymers, are efficient at increasing water diffusion through PDMS. Furthermore, their results indicate that the effect of the fillers seemed to correlate with their water binding capacity, leading to faster water transport through the PDMS film. However, this study did not investigate if addition of the fillers affects the replication capability of hybrid PDMS, which is important for producing replica leaves that sufficiently mimic the living leaf topography.

Thus, we tested the ability of PDMS enriched with 5\% w/w Carbopol or Pemulen to replicate plant leaf surfaces, by using the pillar test pattern $(5 \mu \mathrm{m}$ high, $15 \mu \mathrm{m}$ high, see Fig. 1(a)) in comparison to PDMS Fig. 1(c)). The dimensions of the pattern were chosen to be comparable to structures found on the abaxial (lower) side of the $A$. thaliana leaf, such as stomata or grooves. This side of the leaf more abundantly colonised with microorganisms than the upper surface (adaxial). ${ }^{1)}$ Initially, we attempted to replicate the pattern using a PDMS double-casting protocol which involves a treatment of the intermediate PDMS template, as shown in Fig. 1(b), with 0.1\% w/v HPMC solution as an anti-adhesive agent ${ }^{24,25)}$. This protocol enables PDMS to be effectively peeled away from the intermediate template, or leaf imprint with good fidelity; ${ }^{21)}$ however, for hybrid PDMS the replication quality was not suitable. We repeatedly observed difficulties with the hybrid PDMS penetrating the wells in the intermediate PDMS template. In addition, the hybrid PDMS would remain attached to 
the intermediate PDMS template. The latter in particular resulted in the majority of pillars missing from the final replica, as shown in Fig. 1(d). Increasing the concentration of HPMC to $0.3 \% \mathrm{w} / \mathrm{v}$ did not increase the replication quality.

As a result, we applied an alternative anti-adhesive coating onto the template surface in form of treatment with oxygen plasma followed by exposure to trichloro $(1 \mathrm{H}, 1 \mathrm{H}, 2 \mathrm{H}, 2 \mathrm{H}-$ perfluorooctyl)silane. ${ }^{26,27)}$ Changing the anti-adhesive coating drastically improved the quality of the hybrid PDMS replicas, as demonstrated by Fig. 1(e) and (f). Moreover, this type of coating did not have to be repeated prior to consecutive replication processes. In addition, we compared the dimensions of the replicated PDMS pillars with those made of hybrid PDMS after acquiring their optical profiles. Table 1 compares the average heights and widths of 20 pillars on the photoresist master, the intermediate PDMS template, the PDMS replica, as well as replicas using PDMS + Carbopol and PDMS + Pemulen. Taking into consideration that PDMS shrinks during curing ${ }^{35)}$, the dimensions of pillars on all three replicas are comparable, thus indicating that the addition of the fillers has negligible impact on the replication fidelity of the hybrid PDMS replicas.

\subsection{Replication of leaf surfaces using hybrid PDMS}

As a result of the patterning study using the regular pillar structures, we proceeded to replicating the abaxial side of the A. thaliana leaf. Living leaves, such as our model leaf, represent a significantly more complex replication challenge, due to the variety of surface features found on the leaves and their inherent fragility. This complexity of the leaf surface makes it difficult to perform direct comparative measurements; thus, the need to initially compare the replication of surfaces with regular structures, as demonstrated above. To perform leaf surface replications we repeated the same process, shown in detail in Fig. 2(af), consecutively using the leaf imprints for all of the replica materials. Samples of the living leaves were first taken from the whole leaf(see Fig. 2(a)) and then mounted onto Petri dishes using double-sided tape to allow for PDMS casting (see Fig. 2(b) \&(c)). Once the intermediate leaf imprint was formed in PDMS and treated with anti-adhesion coating (see Fig. 2(d)), it could be repeatedly re-used to replicate into PDMS or hybrid PDMS, as shown in Fig. 2(e) and (f).

For analysis we compared the replica leaves prepared with the hybrid PDMS and PDMS. Due to the surface properties of the replica leaves, in particular the hybrid PDMS, we were unable to obtain high-resolution optical profilometry images as for the regular pattern replication. The white light interferometry technique used to characterize the pillar samples, appeared to be negatively influenced by the difference in reflective properties between 
PDMS and the hybrid PDMS. Instead, we acquired bright field optical microscopy images of the same field of view for PDMS, PDMS + Carbopol and PDMS + Pemulen leaf replica surfaces, as shown in Fig. 2(g-i). The hybrid PDMS shown in Figs. (h) and (i) contained opaque grains in the bulk of the material, which distorted the light pathway when using the light source at the bottom of the sample. These opaque areas may also explain the inability of optical profilometry to obtain a surface scan. Applying a light source located above the sample, however, enabled the surface of the hybrid PDMS replicas to be visualised. The images obtained with this method confirmed the high details of replicas produced in PDMS and both hybrid PDMS polymer blends, indicated by the presence of grooves and stomata in all replicas.

\subsection{Contact angle measurements}

Besides the surface topography, one other key characteristics of plant leaf surfaces is their hydrophobicity. The hydrophobicity of the surface can be determined by measuring the contact angle of water droplets placed onto the surface. A contact angle of $<90^{\circ}$ refers to a hydrophilic surface, $>90^{\circ}$ to a hydrophobic surface, and $>150^{\circ}$ characterizes a superhydrophobic surface. ${ }^{18,36)}$ The hydrophobicity of the surface of plant leaves is a result of a waxy cuticle covering the leaf. ${ }^{37,38)}$ This cuticle is a physical barrier to multiple external factors (e.g. plant pathogens), while its main function is the prevention of water, ion and nutrient loss to the leaf surface. ${ }^{13,39-41)}$ Moreover, the hydrophobicity of the cuticle impacts on microbial attachment on leaf surfaces, which can be observed in form of biofilm formation. ${ }^{4,9)}$

To assess the hydrophobicity of the PDMS and hybrid PDMS abaxial replica leaf surfaces, we measured the contact angles of deionized water droplets that had a volume of less than $80 \mu \mathrm{L}$. We compared these results with the contact angle values acquired for the abaxial surface of $A$. thaliana leaves. As demonstrated in Fig. 3, we observed no significant difference in contact angles between $A$. thaliana leaves $\left(98 \pm 1^{\circ}(\mathrm{SEM}, \mathrm{N}=5)\right)$, and PDMS $\left(97 \pm 1^{\circ}(\mathrm{N}=5)\right)$, PDMS and PDMS + Carbopol $(99 \pm 2(\mathrm{~N}=5)),+$ PDMS + Pemulen replica $(99 \pm 2(\mathrm{~N}=5))$ leaves. Results are in agreeance with contact angles reported in our previous work, which compared the contact angles of PDMS to agar, gelatin, and isolated leaf cuticles. ${ }^{21,22)}$ Due to all of the replica leaf surfaces being hydrophobic, this indicates that their applicability for microbiological studies is promising.

\subsection{Water permeability of PDMS and hybrid PDMS membranes}

As explained above, permeation of water from the plant interior to the leaf surface is limited by the cuticle. The hydrophobicity of the cuticle surface also influences water 
distribution. ${ }^{4,42)}$ To support bacterial life on replica leaves it is important to be able to deliver water and water-soluble molecules to the surface of a replica leaf, in comparable amounts as it occurs in nature. As PDMS is hydrophobic, the water diffusion through the polymer is limited, as demonstrated in Fig. 4(a). The superabsorbent polymers Carbopol and Pemulen were previously shown to increase the permeability of the PDMS to water and inorganic salts. $^{23,43)}$ However, this was tested for silicon films of different formulation, such as RTV-2 silicone gel and Silpuran, and not the Sylgard 184 version we use. Sylgard 184 is commonly used for fabrication microfluidic devices for biological applications, and for bioimprinting. Therefore, we assessed the diffusion of water through $150 \mu \mathrm{m}$ thick PDMS and hybrid PDMS films made from Sylgard 184. The hybrid PDMS films contained the aforementioned superabsorbent polymers at a concentration of $5 \% \mathrm{w} / \mathrm{w}$. We used the experimental setup previously described by Remus-Emsermann et al., ${ }^{13)}$ and measured the water loss through the membranes over time. The water diffusion rates through the hybrid PDMS membranes was significantly higher than those measured for the PDMS membranes, as can be observed in Fig. 4. After the first $24 \mathrm{~h}$, the diffusion of water, and thus the mass flux (see Fig. 4(b)) through the PDMS + Carbopol membranes, were consistently higher than both PDMS and PDMS + Pemulen. In the first 24 hours, PDMS + Pemulen showed a marginally higher water diffusion and mass flux, which is consistent with observations by Borde et al., for the same fillers mixed in RTV-2 silicone. ${ }^{23)}$ While their measurements indicated superior water evaporation of a Pemulen-based silicone hybrid, their experiment was limited to an eight hour period as compared to the $144 \mathrm{~h}$ in total in our case.

In summary, our results demonstrate that Sylgard 184 PDMS, containing the superabsorbent filler Carbopol, has the potential to improve the delivery of water, and watersoluble molecules to the surface of replica leaves - over the time periods relevant to phyllosphere microbiology. The permeability values acquired for $150 \mu \mathrm{m}$ thick membranes were $10^{3}-10^{5}$ times higher, when compared to the literature values measured for isolated leaf cuticles of different plant species. ${ }^{42,44,45)}$ However, the local supply of water cannot be precisely determined at the single bacterial cell level by the diffusion experiments and there is no permeability data for the A. thaliana, as it is impossible to isolate its leaf cuticles. Due to $A$. thaliana leaf properties, we expect higher permeability of water to the surface compared to literature values for other plant species. Moreover, during the diffusion experiments, samples were incubated in a different relative humidity to the one that will be present during observation of bacterial behaviour on the surface. The reason being is that the relative humidity conditions applied during the diffusion experiment, while allowing more precise 
weight loss measurements, may heavily impair the growth of the bacteria. To be able to precisely adjust the amount of water that can diffuse to the surface a different approach needs to be applied. In the future we will use whole cell bacterial bioreporters equipped with a specific genetic tool, which have been shown to sense and report the different levels of water availability on bean leaves ${ }^{46}$, to adjust the permeability of PDMS to values observed in vivo on $A$. thaliana leaves.

\subsection{Visualisation of bacteria on replica surfaces}

Finally, in order to assess if the addition of fillers influences the distribution of microorganisms on the surface, we used a model strain for phyllosphere microbiology, Pantoea agglomerans 299R. ${ }^{31)}$ The strain P. agglomerans $299 \mathrm{R}$ is widely used in phyllosphere microbiology, as it has a fully sequenced genome, has established growth protocols, and can be easily genetically modified. ${ }^{13,31)}$ To assess bacterial distribution we compared the arrangement of bacteria on PDMS and hybrid PDMS replica abaxial leaf surfaces, to those on the abaxial surface of an A. thaliana leaves. For easy tracking of the bacteria on the surface a specific sub-strain P. agglomerans 299R::MRE-Tn7-145, which carries a genomic insertion of Tn-7 transposon containing mScartlet-I protein ${ }^{32)}$ was used. As such, P. agglomerans 299R::MRE-Tn7-145 was spray inoculated onto the replica surfaces and the spray pattern observed using an overlay of bright field and fluorescence microscopy, as shown in Fig. 5. Bacteria distribution patterns observed on the PDMS replicas were similar to those on living A.thaliana leaves. Furthermore, the bacteria distribution patterns observed on the hybrid PDMS surfaces were also similar. However, we observed that the patterns appeared to be influenced by the increased wettability of the hybrid PDMS. This occurred on evenly wetted surfaces and irrespective of whether Carbopol or Pemulen were present as fillers.

The above constitutes the first combined reproduction of microscopic topography and macroscopic wettability in an artificial platform for replica leaves. This is of importance as the levels of success among bacteria of the same genetic background in colonizing a niche can differ on single cell level. ${ }^{47)}$ The microscopic topography of the leaves alone is complex and creates distinguishable microhabitats preferred by microorganisms. By mimicking the topography with leaf replicas it will be possible to investigate bacterial colonizers without biochemical bias, as the composition of substances diffused to the surface will be known. Moreover the topographical structures can work as physical barriers for bacterial community expansion, thus shaping the final outcome. Simultaneously, the importance of macroscale wettability lies in its influence on water and the distribution of water-soluble particles, as 
well as the way the bacteria can interact with the surface. The ideal leaf replica material does not interfere with the bacterial survival, while imitating as many properties of the leaf surface as possible. As the leaf cuticle works as a physical barrier guarding the plant interior, there are limited interactions between bacteria and individual groups on the surface ${ }^{48)}$, with PDMS influencing the bacterial survival in a similar manner as hydrocarbon polymers of the leaf cuticle. $^{21)}$

\section{Conclusions}

Our work demonstrates the potential of hybrid PDMS enriched with superabsorbent polymers, for phyllosphere microbiology. We were able to replicate the three dimensional, heterogeneous topography of the $A$. thaliana leaf surface, using hybrid PDMS. The surface energy of hybrid PDMS replica leaves closely matched the results obtained for the living leaf. Superabsorbent polymers increased the diffusion of water through PDMS, and P. agglomerans 299R::MRE-Tn7-145 formed similar patterns on the PDMS and hybrid PDMS replicas compared to the $A$. thaliana leaf. The results presented in this paper indicate that the hybrid PDMS replica leaves, have the potential to be applied in phyllosphere microbiology studies. In the future, hybrid PDMS replica leaves will be used to study bacterial behaviour, and microbe-microbe interactions.

\section{Acknowledgments}

This work was funded by the Biomolecular Interaction Centre of the University of Canterbury and Marsden Grant UOC1704 awarded to M.R.-E. and V.N. M.B. was supported by a University of Canterbury Doctoral Scholarship and a Biomolecular Interaction Centre doctoral scholarship. R.S. was supported by a Post-Doctoral Fellowship by the National Science Challenge - Science for Technological Innovation. We thank Helen Devereux, Gary Turner and Thomas Evans for technical assistance. 


\section{References}

1) J. A. Vorholt, Nature Rev. Microbiol. 10 (12), 828-40 (2012).

2) Y. Bai, D. B. Müller, G. Srinivas, R. Garrido-Oter, E. Potthoff, M. Rott, N. Dombrowski, P. C. Munch, S. Spaepen, M. Remus-Emsermann, B. Hüttel, A. C. McHardy, J. A. Vorholt, and P. Schulze-Lefert, Nature 528 (7582), 364-69 (2015).

3) K. M. Meyer, and J. H. J. Leveau. Oecologia 168 (3), 621-29 (2011).

4) H. K. Doan, and J. H. J. Leveau. Phytopathology 105 (8), 1036-42 (2015).

5) W. A. Corpe, J. Microbiol. Methods 3 (3-4), 215-21 (1985).

6) G. L. Ercolani, Microb. Ecol. 21 (1), 35-48 (1991).

7) C. Leben, Phytopathology 78 (2), 179 (1988).

8) M. Q. Carter, K. Xue, M. T. Brandl, F. Liu, L. Wu, J. W. Louie, R. E. Mandrell, and J. Zhou. PloS One 7 (9), e44186 (2012).

9) C. E. Morris, and J.-M. Monier. Annu. Rev. Phytopathol. 41, 429-53 (2003).

10) L. Rivas, N. Fegan, and G. A. Dykes. Int. J. Food Microbiol 115 (1), 89-94 (2007).

11) G. A. Beattie, and L. M. Marcell. Phytopathology 92 (9), 1015-23 (2002).

12) J. H. Leveau, and S. E. Lindow. PNAS 98 (6), 3446-53 (2001).

13) M. N. P. Remus-Emsermann, S. de Oliveira, L. Schreiber, and J. H. J. Leveau. Front. Microbiol. 2, 197 (2011).

14) B. Sirinutsomboon, M. J. Delwiche, and G. M. Young. Biosys. Eng. 108 (3), 244-52 (2011).

15) H.-H. Chou, A. Nguyen, A. Chortos, J. W. F. To, C. Lu, J. Mei, T. Kurosawa, W.-G. Bae, J. B-H Tok, and Z. Bao. Nature Comm. 6, 8011 (2015).

16) T. Nakata, H. Liu, Y. Tanaka, N. Nishihashi, X. Wang, and A. Sato. Bioinspiration Biomim. 6 (4), 045002 (2011).

17) S. Nishimoto, and B. Bhushan. RSC Adv. 3, 671-690 (2013).

18) M. Sun, C. Luo, L. Xu, H. Ji, Q. Ouyang, D. Yu, and Y. Chen. Langmuir 21 (19), 8978-81 (2005).

19) B. Wang, W. Liang, Z. Guo, and W. Liu. Chem. Soc. Rev. 44 (1), 336-61 (2015).

20) B. Zhang, Y. Luo, A. J. Pearlstein, J. Aplin, Y. Liu, G. R. Bauchan, G. F. Payne, Q. Wang, X. Nou, and P. D. Millner. ACS Appl. Mat. Interfaces. 6 (15), 12467-78 (2014).

21) R. Soffe, N. Altenhuber, M. Bernach, M. N. P. Remus-Emsermann, and V. Nock. engRxiv 2pzrv (2018).

22) R. Soffe, M. Bernach, M. N. P. Remus-Emsermann, and V. Nock. bioRxiv 523985 (2019).

23) A. Borde, M. Larsson, Y. Odelberg, J. Hagman, P. Löwenhielm, and A. Larsson. Acta Biomat. 8 (2), 579-88 (2012).

24) L. Gitlin, P. Schulze, and D. Belder. Lab Chip 9 (20), 3000-3002 (2009).

25) L. Yang, X. Hao, C. Wang, B. Zhang, and W. Wang. Microsys. Technol. 20 (10-11): 193340 (2013).

26) B. Bhushan, D. Hansford, and K. Kug Lee. JVST A. 24 (4), 1197-1202 (2006).

27) G. Zhuang, and J. P. Kutter. J. Micromechanics Microengineering 21 (10), 105020 (2011).

28) J. He, M. Mao, Y. Liu, J. Shao, Z. Jin, and D. Li. Adv. Healthcare Mat. 2 (8), 1108-13 (2013).

29) A. Tayagui, Y. Sun, D. A. Collings, A. Garrill, and V. Nock. Lab Chip 17 (21), 3643-3653 (2017).

30) J. Schindelin, I. Arganda-Carreras, E. Frise, V. Kaynig, M. Longair, T. Pietzsch, S. Preibisch, et al. Nature Meth. 9 (7), 676-82 (2012).

31) M. N. P. Remus-Emsermann, E. Bae Kim, M. L. Marco, R. Tecon, and J. H. J. Leveau. Genome Announc. 1 (1), e00036-13 (2013).

32) R. O. Schlechter, H. Jun, M. Bernach, S. Oso, E. F. J. Boyd, D. A. Muñoz-Lintz, R. C. J. Dobson, D. M. Remus, and M. N. P. Remus-Emsermann. bioRxiv. 402172 (2018).

33) M. N. P. Remus-Emsermann, R. Tecon, G. A. Kowalchuk, and J. H. J. Leveau. ISME J. 6 (4), 756-65 (2012).

34) G. A. Beattie, and S. E. Lindow. Phytopathology 89 (5), 353-59 (1999).

35) S. W. Lee, and S. S. Lee, Microsyst. Technol. 14, 205 (2008).

36) Y. Yuan, and T. R. Lee. In: G. Bracco, and B. Holst, Eds., Surface Science Techniques (Springer 2013) Volume 51, pp. 3-34.

37) P. E. Kolattukudy, Annu. Rev. Plant Physiol. Plant Mol. Biol. 32, 539-567 (1981).

38) L. Schreiber. Trends Plant Sci. 15 (10), 546-553 (2010).

39) J. Schönherr, and L. Schreiber. Planta 219 (3), 405-411 (2004). 
40) L. Schreiber, S. Elshatshat, K. Koch, J. Lin and J. Santrucek. Planta 223 (2), 283-290 (2006).

41) W. Aragón, J. J. Reina-Pinto and M. Serrano. J. Exp. Bot. 68 (19), 5339-5350 (2017).

42) L. Schreiber, and J. Schonherr. Water and Solute Permeability of Plant Cuticles (Springer 2009).

43) P. Lopour, and V. Janatova. Biomaterials 16 (8), 633-640 (1995).

44) L. Schreiber and M. Riederer. Oecologia 107, 426-432 (1996).

45) L. Schreiber and M. Riederer. Plant Cell Environ. 19, 1075-1082 (1996).

46) C. A. Axtell and G. A. Beattie. Appl. Environ. Microbiol. 68, 4604-4612 (2002).

47) S. V. Avery. Nat. Rev. Micro. 4, 577 (2006).

48) T. H. Yeats and J. K. C. Rose. Plant Physiol. 163, 5-20 (2013).

Table 1. Pattern Resolution Measurements. Height and width measurements were taken from 20 pillars or wells in case of the intermediate PDMS template. All data is represented as mean \pm standard deviation. Standard deviation has been used to show the scattering in measured values.

\begin{tabular}{lccccc}
\hline & $\begin{array}{c}\text { Photoresist } \\
\text { Mold } \\
\text { Master }\end{array}$ & $\begin{array}{c}\text { Intermediate } \\
\text { PDMS } \\
\text { Template }\end{array}$ & $\begin{array}{c}\text { Patterned } \\
\text { PDMS } \\
\text { (silane) }\end{array}$ & $\begin{array}{c}\text { Patterned } \\
\text { PDMS-Carbopol } \\
\text { (silane) }\end{array}$ & $\begin{array}{c}\text { Patterned } \\
\text { PDMS-Pemulen } \\
\text { (silane) }\end{array}$ \\
\hline $\begin{array}{l}\text { Height } \\
(\boldsymbol{\mu m})\end{array}$ & $4.50 \pm 0.03$ & $4.18 \pm 0.01$ & $4.71 \pm 0.01$ & $4.37 \pm 0.04$ & $4.33 \pm 0.03$ \\
$\begin{array}{l}\text { Width } \\
(\boldsymbol{\mu m})\end{array}$ & $14.94 \pm 0.21$ & $15.11 \pm 0.52$ & $\begin{array}{c}14.00 \pm \\
0.38\end{array}$ & $14.52 \pm 0.24$ & $14.55 \pm 0.23$ \\
\hline
\end{tabular}

\section{Figure Captions}

Fig. 1. Optical profilometry images demonstrating the fidelity of pattern transfer from a (a) ADEX photoresist mold master to (b) the PDMS intermediate template, and (c) patterned PDMS, (d) patterned PDMS + 5\% Carbopol (HPMC), (e) patterned PDMS + 5\% Carbopol (silane), and (f) patterned PDMS + 5\% Pemulen (silane) replicas. Note, only a small section of the overall pattern is shown for clarity. All optical profilometer images were filtered using a 3-point level function in Profilm3D software (Filmetrics). As can be observed from (d), anti-adhesion treatment with HPMC lead to loss of detail, due to insufficient detachment from the intermediate PDMS template.

Fig. 2. Schematic of the replication process used to fabricate hybrid PDMS replica leaves and optical microscopy images showing the replication fidelity. Samples were cut out of living $A$. thaliana leaves (a) and affixed on a Petri dish. PDMS at 20:1 w/w ratio was cast onto the leaf sample (c), cured and peeled off to produce an intermediate PDMS leaf imprint (d). This imprint was treated for anti-adhesion and casted with PDMS or hybrid PDMS (e) 
to produce the replica leaf surfaces. Optical bright field microscopy images of (g) PDMS, (h) PDMS + 5\% Carbopol, and (i) PDMS + 5\% Pemulen replica leaves. The images represent the same location on the replicas fabricated using the same leaf imprint. As can be observed, Carbopol and Pemulen fillers used to produce the hybrid PDMS show up as dark areas in (h) and (i), which may affect the suitability of these hybrids for microscopy.

Fig. 3. Contact angle measurements of living abaxial (lower) $A$. thaliana leaves, PDMS and hybrid PDMS replica abaxial leaf surfaces. Results are presented as mean $\pm \mathrm{SEM}$ (standard error mean). N.S. indicates no significant difference between the measurements. As can be observed, contact angles of PDMS and Carbopol and Pemulen-containing PDMS hybrids are comparable to that of living $A$. thaliana leaves. This indicates that addition of the fillers does not significantly alter the surface energy of PDMS, thus making the hybrid PDMS replicas a suitable alternative to PDMS replica leaves, for phyllosphere microbiology studies.

Fig. 4. Diffusion of water through PDMS and hybrid PDMS membranes. (a) Graph of the amount of water diffused through $150 \mu \mathrm{m}$ thick PDMS, PDMS + 5\% Carbopol and PDMS + $5 \%$ Pemulen over time. Aluminium film was used as control. As can be observed, after $24 \mathrm{~h}$ the hybrid PDMS membranes exhibited an increased water diffusion compared to PDMS. PDMS $+5 \%$ Carbopol consistently showing the highest diffusion. (b) Plot of the same data as mass flux over time. While PDMS + 5\% Pemulen initially shows a higher mass flux, PDMS: $1+5 \%$ Carbopol outperforms both it and PDMS after about $48 \mathrm{~h}$ up to the total $144 \mathrm{~h}$ recorded.

Fig. 5. Bacterial distribution on abaxial replica surfaces. Overlay of brightfield and fluorescent images visualizing the localization of bacterium $P$. agglomerans 299R::MRETn7-145 on (a) PDMS, (b) PDMS + 5\% Carbopol, and (c) PDMS + 5\% Pemulen replica surface of $A$. thaliana. Distribution of bacteria (green fluorescence) is similar on all three surfaces and comparable to $A$. thaliana (data not shown). However the hybrid PDMS surfaces appeared to be influenced by increased wettability. The images also indicate that fluorescent imaging is possible. Thus, indicating that the replica leaf surfaces are suitable for fluorescence-based bioreporter studies. Scale bars are $100 \mu \mathrm{m}$. 


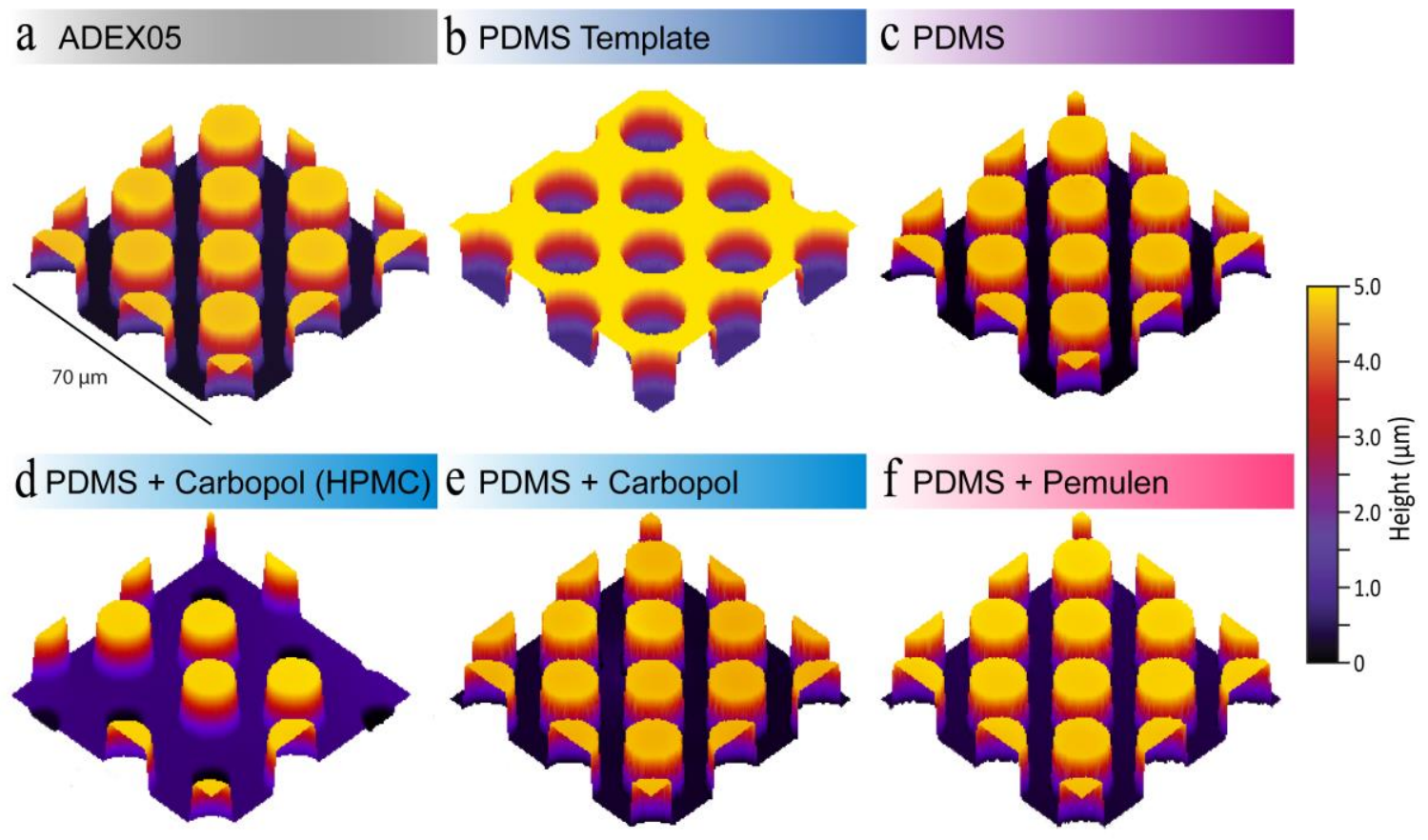

Fig.1. 

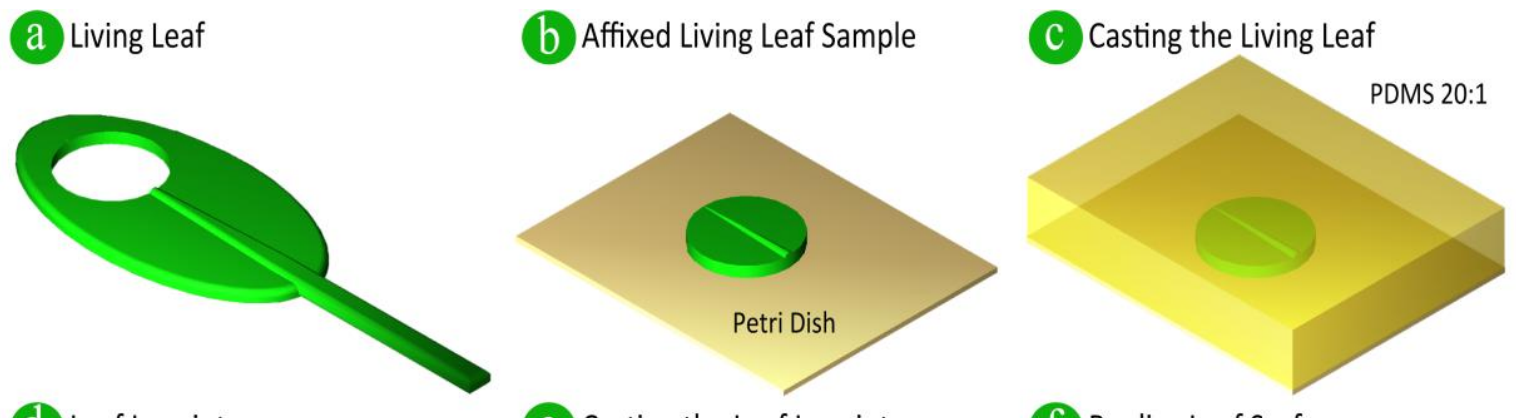

(d) Leaf Imprint
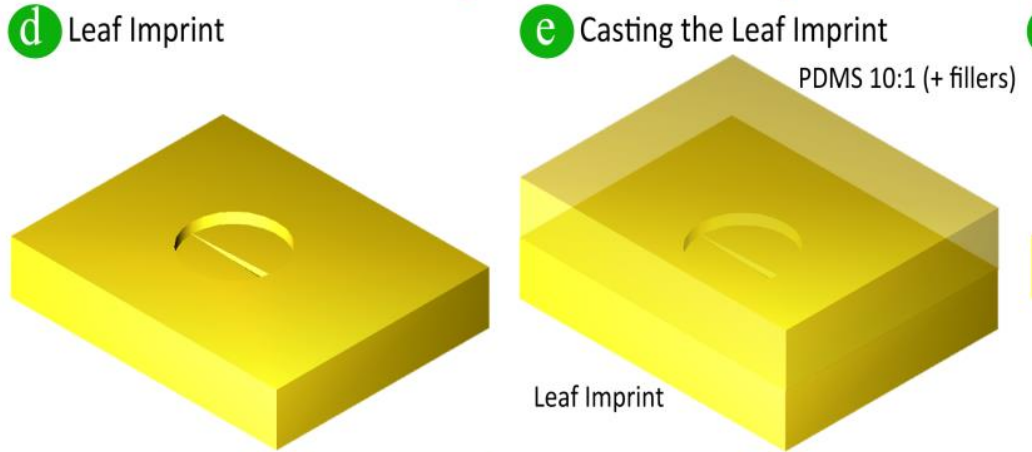

Replica Leaf Surface
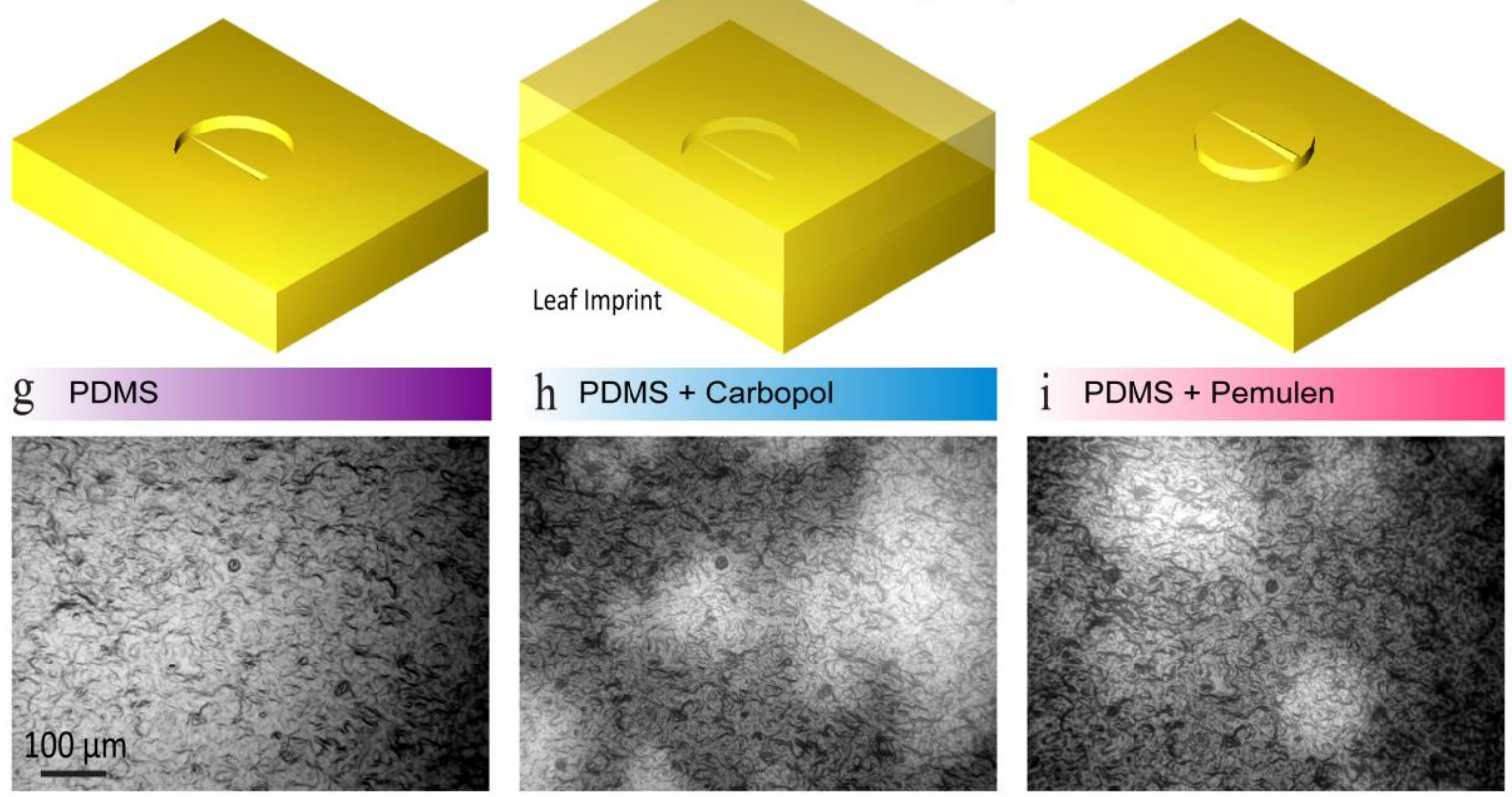

i PDMS + Pemulen

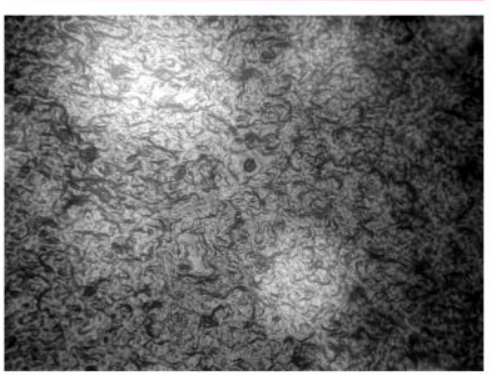

Fig. 2.

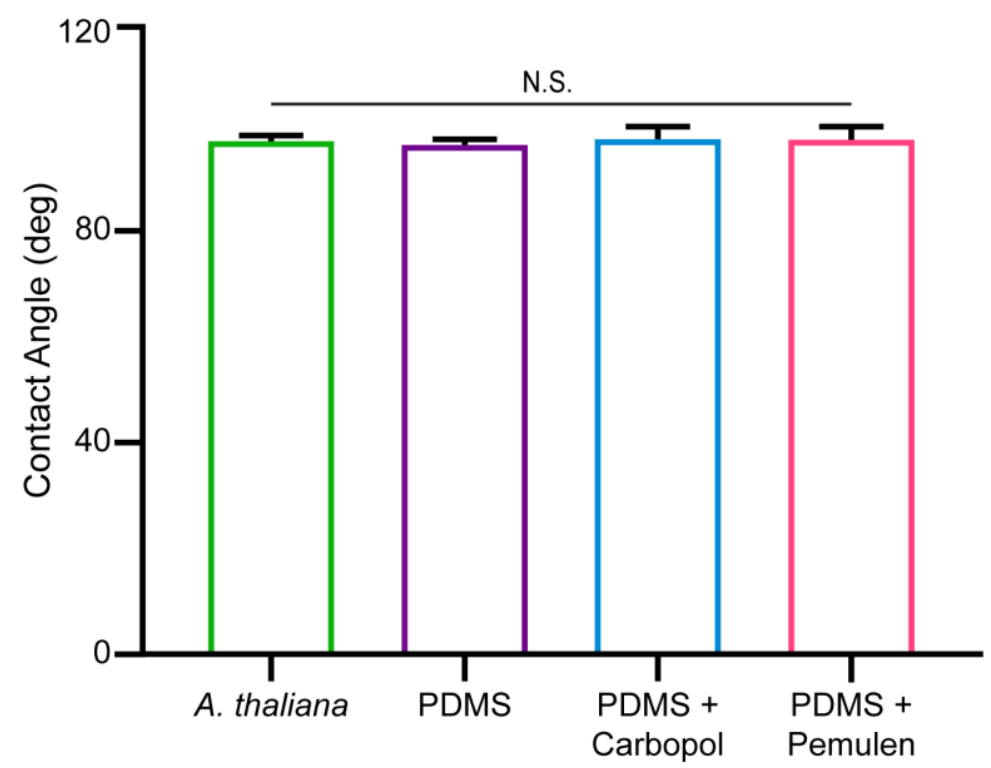

Fig. 3. 

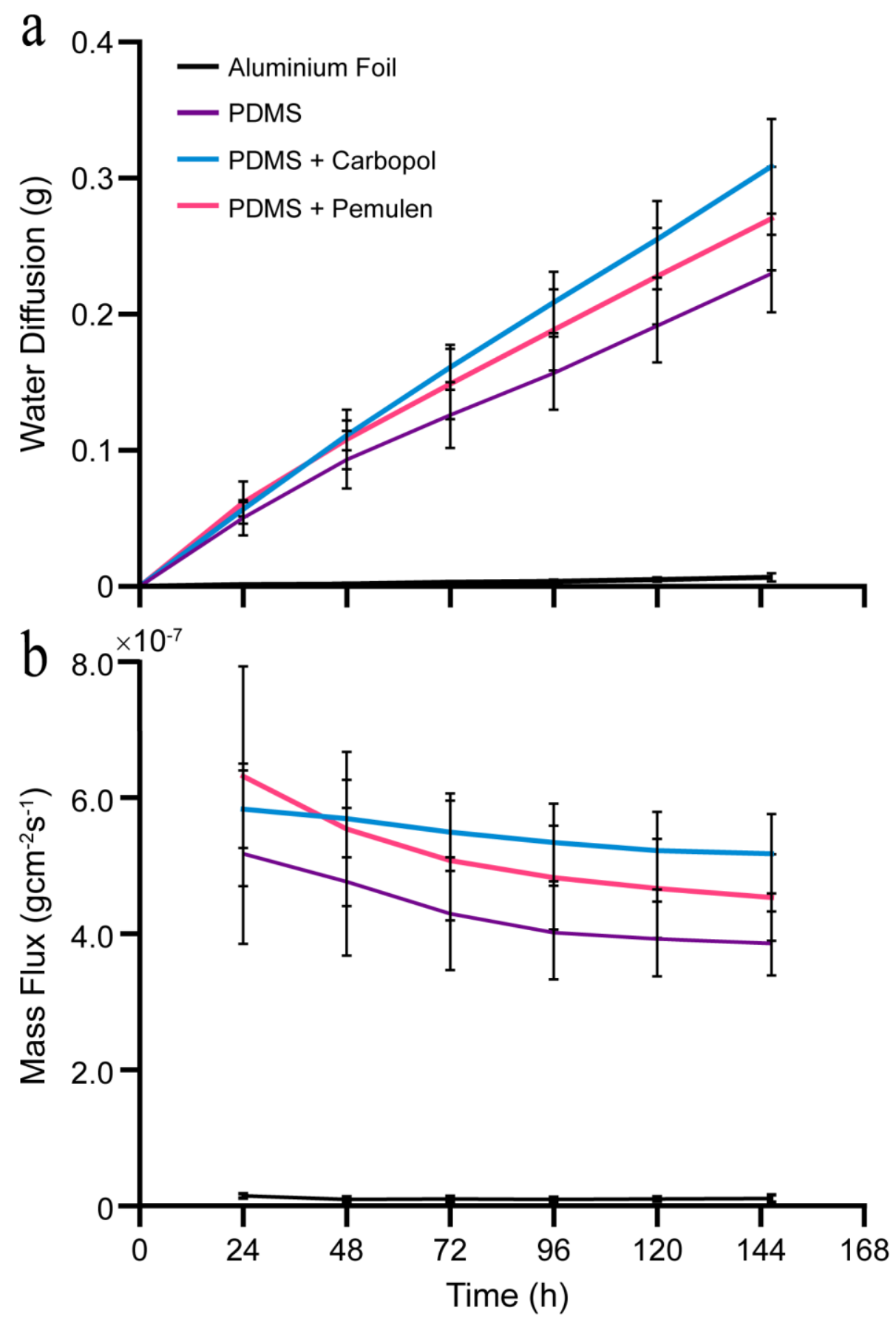

Fig. 4. 

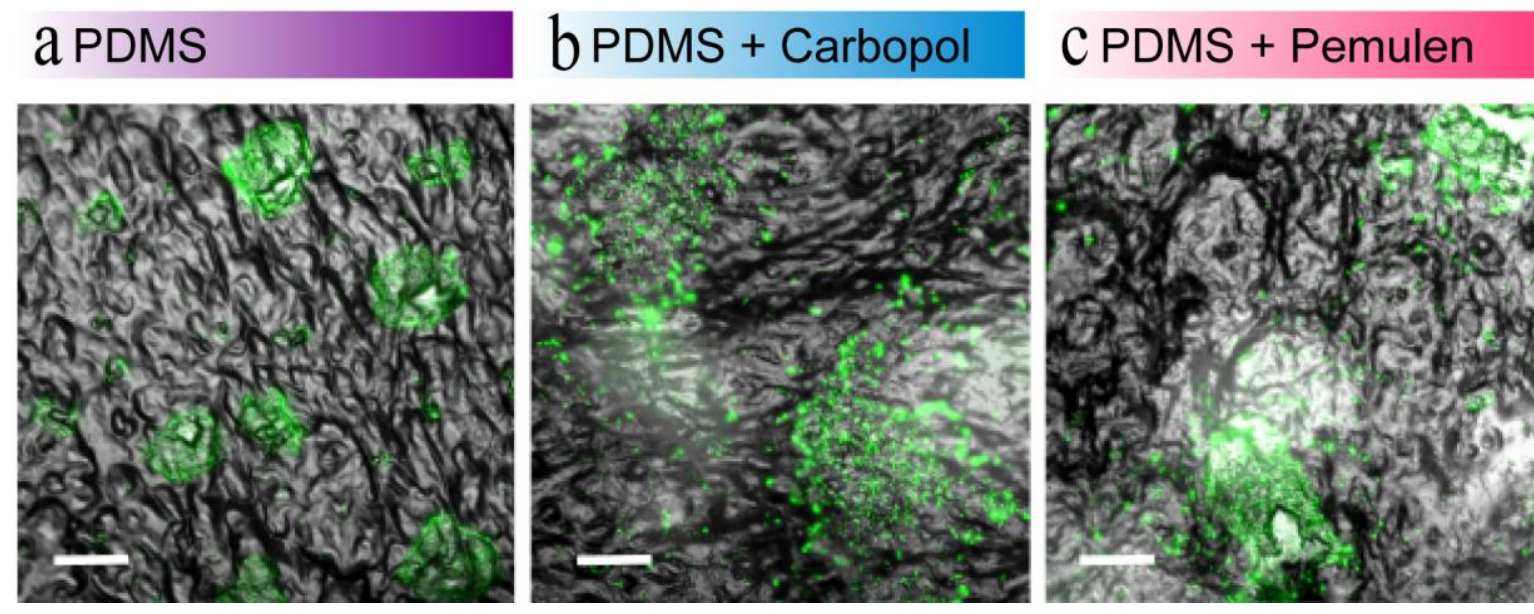

Fig. 5.

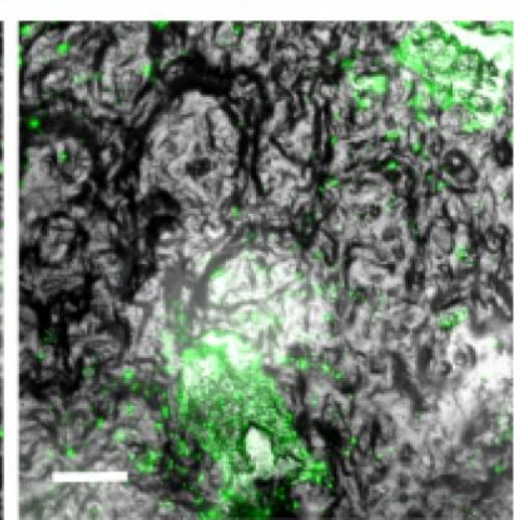

